\title{
Distributed Beamforming and Power Allocation for Cooperative Networks
}

\author{
Zhiguo Ding, Member, IEEE, Woon Hau Chin, Member, IEEE, and Kin K. Leung, Fellow, IEEE
}

\begin{abstract}
Cooperative diversity systems rely on using relay nodes to relay copies of transmitted information to the destination such that each copy experiences different channel fading, hence increasing the diversity of the system. However, without proper processing of the message at the relays, the performance of the cooperative system may not necessarily perform better than direct transmission systems. In this paper, we proposed a distributed beamforming and power allocation algorithm which substantially improves the diversity of the system with only very limited feedback from the destination node. We also derive outage probability as well as study the outage behavior of this scheme.
\end{abstract}

Index Terms-Cooperative networks, distributed beamforming, cooperative diversity, outage probability.

\section{INTRODUCTION}

$\mathbf{O}$ $\mathrm{NE}$ of the challenges of communicating a signal over a medium is the distortion and loss of signal power caused by the medium. Such a phenomenon, commonly termed channel fading, results in fluctuation of the signals received. To combat channel fading, diversity techniques, whereby signals carrying the same information are transmitted and received over different resources, are commonly used. Traditionally, to introduce diversity, the multiple copies of message signal will span time, frequency or spatial resources [1]. A new technique to introduce diversity into the system, in which multiple spatially separated communication devices (nodes) cooperate to improve the quality of communications between two nodes was proposed in [2], [3]. Such systems are able to introduce diversity into the system by using cooperative nodes to relay the information to the destination after some delay. Several protocols to accomplish the task has been introduced in [2], of which, Amplify and Forward (AF) has been shown to achieve full diversity.

Since then, there have been many algorithms proposed to manipulate the relay nodes to enhance performance. Known as cooperative beamforming, most of these algorithms weigh

Manuscript received January 29, 2007; revised June 15, 2007 and August 30, 2007; accepted September 24, 2007. The associate editor coordinating the review of this paper and approving it for publication was C. Xiao. This research was sponsored by US Army Research Laboratory and the UK Ministry of Defence and was accomplished under Agreement Number W911NF-06-3-0001. The views and conclusions contained in this document are those of the authors and should not be interpreted as representing the official policies, either expressed or implied, of the US Army Research Laboratory, the U.S. Government, the UK Ministry of Defense, or the UK Government. The US and UK Governments are authorized to reproduce and distribute reprints for Government purposes notwithstanding any copyright notation hereon.

Z. Ding is with the Department of Communication Systems, Lancaster University, Lancaster, UK, LA1 4WA (e-mail: zhiguo.ding@imperial.ac.uk).

W. H. Chin is with the Institute for Infocomm Research, Singapore 119613 (e-mail: w.h.chin@ieee.org).

K. K. Leung is with the Department of Electrical Engineering, Imperial College, London, SW7 2BT, UK (e-mail: Kin.Leung@imperial.ac.uk).

Digital Object Identifier 10.1109/TWC.2008.070105.

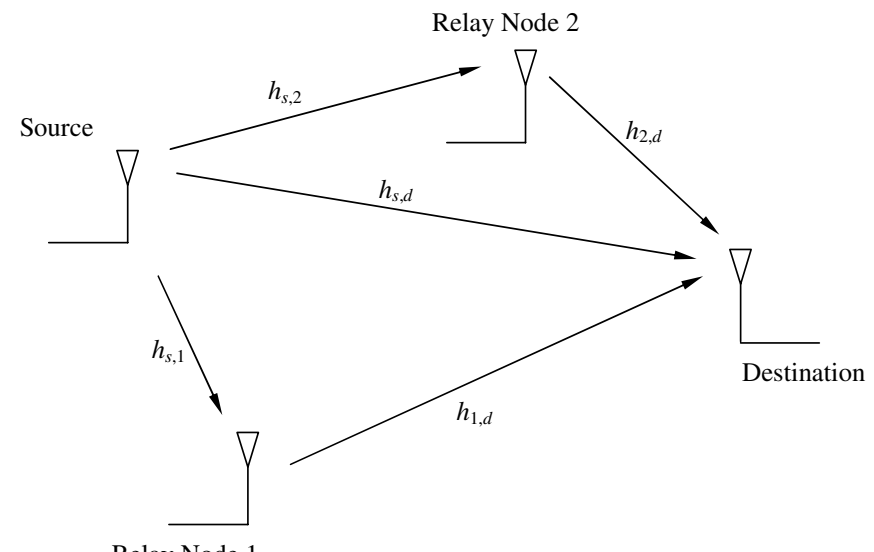

Fig. 1. Cooperative communication system with 2 relay nodes

their input according to the channel state information feedback [4], [5] or prior information available [6]. The optimal weighting for each of the nodes has also been derived in [7]. There have also been algorithms to optimally allocate power to the different relay nodes [8], [9]. At the time of writing this paper, it has also come to our attention of [10], which proposed a scheme similar to ours here. However, the two schemes are based on different scenarios, and the proposed strategies of power allocation in both schemes are different. In this paper, we present a suboptimal distributed beamforming and power allocation scheme which has an outage probability close to the optimal scheme. We further derive the outage probability of the scheme and show that it offers an advantage over schemes where equal weights are assigned to the relays.

\section{SySTEM MODEL}

Consider a cooperative network system with one source node, $N_{r}$ relays nodes, and one destination node as shown in Fig. 1. We assume that the channel state information of incoming and outgoing links are available at each node and that these are flat fading channels. We further assume that the transmission time frame for sending each message is split into two, the first being the message sending by the source node, which we term the "broadcast" phase, and the second being the transmission of the message by the relay nodes, which we refer to as the "cooperation" phase. Here, we consider the non-regenerative relay method.

During the "broadcast" phase, where a signal $x$ is sent by the source node, the received signal at the destination and relay node $i$ can be respectively written as

$$
y_{s, d}=h_{s, d} x+w_{s, d},
$$

and

$$
y_{s, i}=h_{s, i} x+w_{s, i},
$$


where $h_{s, d}$ and $h_{s, i}$ denotes the channel response from the source to the destination node and relay node $i$ respectively, while $w_{s, d}$ and $w_{s, i}$ are the observation noise at the destination and relay node $i$ respectively.

The received signal at the destination node during "cooperation" phase is written as

$$
\begin{aligned}
y_{r, d} & =\sum_{i=1}^{N_{r}} \frac{\alpha_{i}}{\sqrt{\sigma_{n}^{2}+\left|h_{s, i}\right|^{2}}} h_{i, d}\left(h_{s, i} x+w_{s, i}\right)+w_{r, d} \\
& =\left[\begin{array}{lll}
\frac{h_{s, 1} h_{1, d}}{\sqrt{\sigma_{n}^{2}+\left|h_{s, 1}\right|^{2}}} & \ldots & \frac{h_{s, N_{r}} h_{N_{r}, d}}{\sqrt{\sigma_{n}^{2}+\left|h_{s, N_{r}}\right|^{2}}}
\end{array}\right]\left[\begin{array}{c}
\alpha_{1} \\
\vdots \\
\alpha_{N_{r}}
\end{array}\right] x \\
& +\left[\begin{array}{lll}
\frac{w_{s, 1} h_{1, d}}{\sqrt{\sigma_{n}^{2}+\left|h_{s, 1}\right|^{2}}} & \ldots & \frac{w_{s, N_{r} h_{N_{r}, d}}}{\sqrt{\sigma_{n}^{2}+\mid h_{s,\left.N_{r}\right|^{2}}}}
\end{array}\right]\left[\begin{array}{c}
\alpha_{1} \\
\vdots \\
\alpha_{N_{r}}
\end{array}\right]+w_{r, d} \\
& =\mathbf{h f} x+\mathbf{w f}+w_{r, d}
\end{aligned}
$$

where $w_{r, d}$ is the noise at the destination node during the "cooperation" phase, $\sigma_{n}^{2}$ is the variance of the noise at each of the relays, and the factor $\frac{1}{\sqrt{\sigma_{n}^{2}+\left|h_{s, i}\right|^{2}}}$ is due to power normalization at each relay. The vector $\mathbf{f}=\left[\begin{array}{lll}\alpha_{1} & \cdots & \alpha_{N_{r}}\end{array}\right]^{T}$ is the weight vector which we will derive in the following section.

Concatenating equations (1) and (3), the signal model for the relay network using the amplify-forward protocol can be written in matrix form as

$$
\left[\begin{array}{l}
y_{s, d} \\
y_{r, d}
\end{array}\right]=\left[\begin{array}{c}
h_{s, d} \\
\mathbf{h f}
\end{array}\right] x+\left[\begin{array}{c}
w_{s, d} \\
\mathbf{w f}+w_{r, d}
\end{array}\right]
$$

\section{Distributed BeAmForming AND Power AllocAtion}

To maximize the SNR at the destination, the optimal weight vector $\mathbf{f}$ should satisfy

$$
\begin{aligned}
& \max _{\mathbf{f}} E\left\{\frac{P \mathbf{f}^{H} \mathbf{h}^{H} \mathbf{h f}}{\mathbf{f}^{H} \mathbf{w}^{H} \mathbf{w} \mathbf{f}+\left|w_{r, d}\right|^{2}}\right\} \\
& =\max _{\mathbf{f}} E\left\{\frac{P \mathbf{f}^{H} \mathbf{h}^{H} \mathbf{h f}}{\mathbf{f}^{H}\left[\mathbf{w}^{H} \mathbf{w}+\left|w_{r, d}\right|^{2} \mathbf{I}_{N_{r}}\right] \mathbf{f}}\right\},
\end{aligned}
$$

where $P$ is the overall power constraint of the relay nodes.

The optimal solution $\mathbf{f}_{o p t}$ can be found by solving the generalized eigenvalue problem

$$
E\left\{\left[\mathbf{w}^{H} \mathbf{w}+\left|w_{r, d}\right|^{2} \mathbf{I}_{N_{r}}\right]\right\}^{-1} P \mathbf{h}^{H} \mathbf{h f}_{o p t}=\lambda_{\max } \mathbf{f}_{o p t}
$$

where $\lambda_{\max }$ is the largest generalized eigenvalue of $E\left\{\left[\mathbf{w}^{H} \mathbf{w}+\left|w_{r, d}\right|^{2} \mathbf{I}_{N_{r}}\right]\right\}^{-1} P \mathbf{h}^{H} \mathbf{h}$.

However, a centralized control with access to all channel information is required to obtain this optimal solution, and very high system overhead is involved. In this paper, we are interested in a distributed solution, where each relay node can decide its transmit power based on knowledge of its incoming and outgoing channels, $h_{s, i}$ and $h_{i, s}$. Here, we propose to use the weight vector

$$
\mathbf{f}_{p}=\beta\left[\begin{array}{lll}
\frac{h_{s, 1}^{*} h_{1, d}^{*}\left|h_{s, 1}\right|^{2}}{\sqrt{\sigma_{n}^{2}+\left|h_{s, 1}\right|^{2}}} \sqrt{P} & \ldots & \frac{h_{s, N_{r}}^{*} h_{N_{r}, d}^{*}\left|h_{s, N_{r}}\right|^{2}}{\sqrt{\sigma_{n}^{2}+\left|h_{s, N_{r}}\right|^{2}}} \sqrt{P}
\end{array}\right]
$$

where $\beta=1 / \sqrt{\sum_{i=1}^{N_{r}} \frac{\left|h_{s, i}\right|^{6}\left|h_{i, d}\right|^{2}}{\sigma_{n}^{2}+\left|h_{s, i}\right|^{2}}}$ is the normalizing constant such that the power constraint $\mathbf{f}^{H} \mathbf{f}=P$ is satisfied, and the common factor $\beta$ can be periodically obtained from the destination node via a broadcast channel. The reason to introduce a constraint for the total power consumption can be clarified as follows. Although each relay node can transmit up to their own output power limit, the total power constraint limits interference to other source-destination pairs, which is an important issue for power-constrained communication scenarios. The full CSI assumption at the destination can facilitate the calculation of $\beta^{1}$. With such a full CSI assumption, a centralized scheme in (6) can be accomplished, but will still result in more system overhead than the proposed one. The proposed scheme only requires one channel use to broadcast the power constraint to the relays, whereas the centralized one requires at least $N_{r}$ channel uses. Furthermore, the power constraint $\beta$ is only a constant or scaling factor which provides multiple relays a benchmark to calculate their weighting factors. An incorrect $\beta$ means that all relays will operate at a sub-optimal power level. But the relationship between the weighting factors at the multiple relays is still maintained, which is important to prevent signal cancellation among the multiple transmit antennas.

Note that the transmission power of each relay in (7) is proportional to its incoming and outgoing channel coefficients, which fits the intuition that more power should be allocated to relaying nodes with better quality links. The exact expression of the distributed beamformer was obtained from simulation experiments which showed that in (7) achieves the performance close to the optimal solution. To get the insights of such performance superiority, some information-theoretic metrics, such as the outage probability and the diversity-multiplexing tradeoff, will be developed for the proposed beamformer in the next section.

\section{OUtAGE PROBABILITY}

For the proposed distributed beamforming and power allocation scheme, we analyze effects of the scheme on system performance. We focus on outage probability as the information-theoretic measure as in [2].

For the system described by (4), the mutual information is

$$
\mathcal{I}=\frac{1}{2} \log \left[1+\left(\left|h_{s, d}\right|^{2}+\mathbf{f}^{H} \mathbf{h}^{H} \mathbf{h f}\right)\left(E\left\{\mathbf{n}^{H} \mathbf{n}\right\}\right)^{-1}\right]
$$

where $\mathbf{n}=\left[\begin{array}{c}w_{s, d} \\ \mathbf{w} \mathbf{f}+w_{r, d}\end{array}\right]$.

Using the proposed relay weights $\mathbf{f}_{p}$ in (7), we obtain (9), where

$$
\begin{aligned}
E\left\{\mathbf{n}^{H} \mathbf{n}\right\} & =2 \sigma_{n}^{2}+\mathbf{f}^{H} E\left\{\mathbf{w}^{H} \mathbf{w}\right\} \mathbf{f} \\
& =2 \sigma_{n}^{2}+\mathbf{f}^{H} \operatorname{diag}\left\{\frac{\sigma_{n}^{2}\left|h_{1, d}\right|^{2}}{\sigma_{n}^{2}+\left|h_{s, i}\right|^{2}}, \ldots, \frac{\sigma_{n}^{2}\left|h_{N_{r}, d}\right|^{2}}{\sigma_{n}^{2}+\mid h_{s,\left.N_{r}\right|^{2}}}\right\} \mathbf{f} \\
& =\sigma_{n}^{2}\left(2+\frac{P}{\sum_{i=1}^{N_{r}} \frac{\left|h_{s, i}\right|^{6}\left|h_{i, d}\right|^{2}}{\sigma_{n}^{2}+\left|h_{s, i}\right|^{2}}} \sum_{i=1}^{N_{r}} \frac{\left|h_{s, i}\right|^{6}\left|h_{i, d}\right|^{4}}{\left(\sigma_{n}^{2}+\left|h_{s, i}\right|^{2}\right)^{2}}\right) .
\end{aligned}
$$

\footnotetext{
${ }^{1} \mathrm{~A}$ weaker assumption to calculate $\beta$ is that each node will send the realvalued $\frac{\left|h_{s, i}\right|^{6}\left|h_{i, d}\right|^{2}}{\sigma_{n}^{2}+\left|h_{s, i}\right|^{2}}$ to the destination, and hence will consume $N_{r}$ channel
} uses, whereas the full CSI assumption will require $2 N_{r}$ channel uses. 


$$
\begin{aligned}
\mathcal{I} & =\frac{1}{2} \log \left[1+\left(\left|h_{s, d}\right|^{2} P+\mathbf{f}^{H} \mathbf{h}^{H} \mathbf{h} \mathbf{f}\right)\left(E\left\{\mathbf{n}^{H} \mathbf{n}\right\}\right)^{-1}\right] \\
& =\frac{1}{2} \log \left[1+\frac{P}{\sigma_{n}^{2}}\left(\left|h_{s, d}\right|^{2}+\frac{\left(\sum_{i=1}^{N_{r}} \frac{\left|h_{s, i}\right|^{4}\left|h_{i, d}\right|^{2}}{\sigma_{n}^{2}+\left|h_{s, i}\right|^{2}}\right)^{2}}{\sum_{i=1}^{N_{r}} \frac{\left.\left|h_{s, i}\right| h_{i, d}\right|^{2}}{\sigma_{n}^{2}+\left|h_{s, i}\right|^{2}}}\right)\left(2+P \frac{\sum_{i=1}^{N_{r}} \frac{\left|h_{s, i}\right|^{6}\left|h_{i, d}\right|^{4}}{\left(\sigma_{n}^{2}+\left|h_{s, i}\right|^{2}\right)^{2}}}{\sum_{i=1}^{N_{r}} \frac{\left.\left|h_{s, i}\right| h_{i, d}\right|^{2}}{\sigma_{n}^{2}+\left|h_{s, i}\right|^{2}}}\right)^{-1}\right]
\end{aligned}
$$

In this paper, we use the special symbol $\doteq$ to denote exponential equality [11], i.e., $f(\rho) \doteq \rho^{n}$ to denote

$$
\lim _{\rho \rightarrow \infty} \frac{\log f(\rho)}{\log \rho}=n \text {. }
$$

The following theorem describes the high SNR behavior of the proposed distributed beamforming and power allocation algorithm.

Theorem 1: For i.i.d. Rayleigh fading channels and high SNR region, the outage probability of the $N_{r}$-relay system can be approximated as

$$
p(\mathcal{I}<R) \doteq \rho^{-\left(N_{r}+1\right)} .
$$

Proof: The proof is given in the Appendix.

Consider the traditional point-to-point multiple-input singleoutput (MISO) system as a comparable scheme. It is obvious that the performance of such a MISO system is the best that the proposed cooperative strategy can achieve. Provided that there are $N_{r}$ transmit antennas, it can can expected that such a MISO system can achieve $N_{r}+1$ diversity gain since the multiple antennas can coordinate with each other and hence the receiver can have $N_{r}+1$ independent copies of the transmitted signals [1]. Theorem 1 indicates that such diversity gain is still achievable although the multiple antennas are distributed among the multiple nodes. As defined in [11], the multiplexing gain is $r \triangleq \lim _{\rho \rightarrow \infty} \frac{R(\rho)}{\log \rho}$ and the diversity gain is $d \triangleq-\lim _{\rho \rightarrow \infty} \frac{\log p(\mathcal{I}<R)}{\log \rho}$ [11]. From Theorem 1, we have the following corollary.

Corollary 2: The diversity-multiplexing tradeoff achieved by the distributed beamforming and power allocation is given by

$$
d(r)=\left(N_{r}+1\right)(1-2 r) .
$$

Proof: Recall from the proof for Theorem 1, the outage probability is bounded as

$$
\frac{N_{r}(\alpha \theta)^{N_{r}+1}}{\left(N_{r}+1\right) !} \geq p(\mathcal{I}<R) \geq \frac{(\alpha \theta)^{N_{r}+1}}{\left(N_{r}+1\right) !}
$$

where $\theta=\frac{2^{2 R}-1}{\rho}$.

To obtain the diversity-multiplexing tradeoff of the proposed distributed beamforming scheme, define the data rate $R$ as a function of $\rho$ as

$$
R=r \log \rho .
$$

Substitute (13) into the expression of the outage probability and we have

$$
\begin{gathered}
\frac{N_{r}(\alpha)^{N_{r}+1}}{\left(N_{r}+1\right) !} \frac{\left(2^{2 r \log \rho}-1\right)^{N_{r}+1}}{\rho^{N_{r}+1}} \geq p(\mathcal{I}<R) \\
\geq \frac{(\alpha)^{N_{r}+1}}{\left(N_{r}+1\right) !} \frac{\left(2^{2 r \log \rho}-1\right)^{N_{r}+1}}{\rho^{N_{r}+1}}
\end{gathered}
$$

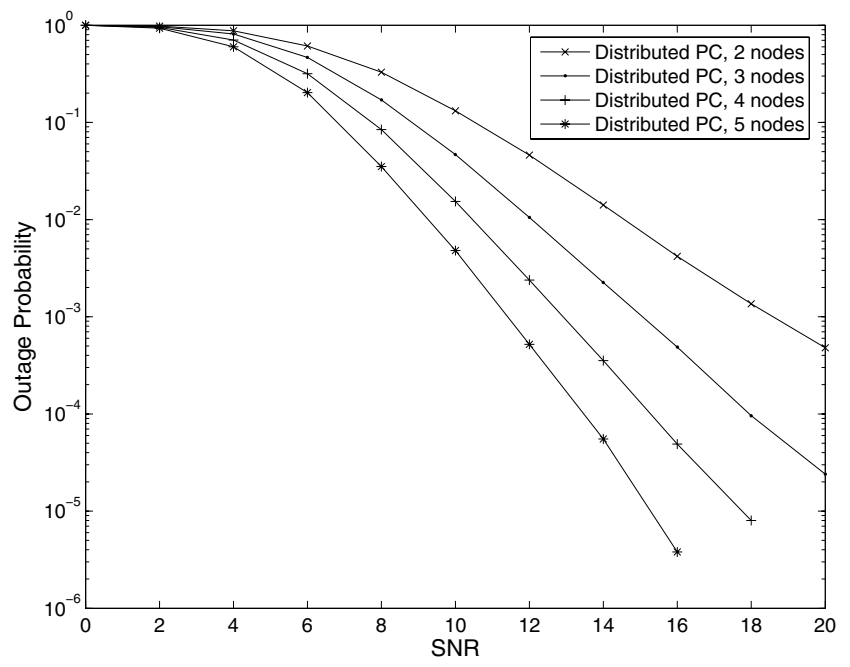

Fig. 2. The outage probability of the proposed beamforming and power allocation schemes with different number of relay nodes versus SNR.

When $\rho \rightarrow \infty$, we have

$$
\log p(\mathcal{I}<R)=\log \left(\frac{\left(\rho^{2 r}\right)^{N_{r}+1}}{\rho^{N_{r}+1}}\right) .
$$

And the corollary is proved.

It is important to point out that although similar results have been obtained previously (e.g., [11], [12]), our situation is unique. Provided that all antennas are located at the source node, the results on full diversity order and the multiplexingdiversity tradeoff could be easily obtained. However, with multiple antennas scattering among the multiple nodes, Theorem 1 and Corollary 2 are valuable as they indicate that the single-antenna source node can function as if it is equipped with multiple antennas. However, it is worthy to point out that such diversity gain is obtained with the price of reducing multiplexing gain, which is caused by the fact that the relaying transmission requires the extra use of bandwidth resources. If complex radio hardware is not available, the opportunistic relaying protocol can be an alternative for distributed phasedarrays. As shown in [12] such an opportunistic scheme can yield the same diversity order as the proposed beamformer.

\section{NumericAl Results}

Consider a relay ad-hoc network where each node is equipped with one antenna element. The wireless links are assumed as flat Raleigh fading. The required data rate is set as $R=1$.

Figure 2 shows the outage performance of the proposed distributed beamforming and power allocation scheme as a function of SNR, where the effect of different number of 


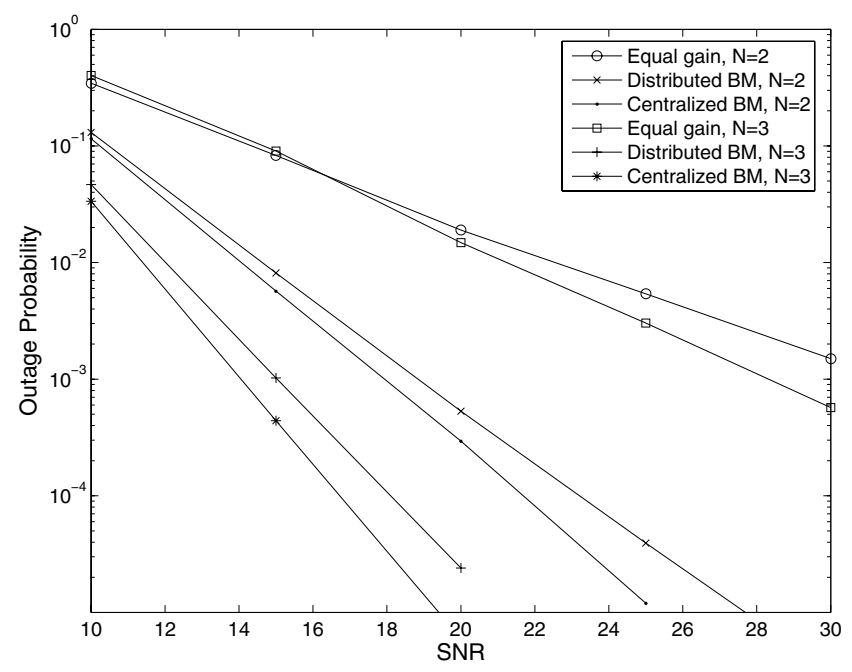

Fig. 3. The outage probability of the various beamforming schemes versus SNR.

relay nodes is also shown. As can be seen from the figure, by increasing the number of the used relay nodes, the proposed method can improve the system performance generally.

In Figure 3 the outage probability of the different beamforming schemes is shown, obtained by Monte Carlo simulations. The two-relay-node scenario, $N_{r}=2$, is considered here. The performance of two existing schemes is also shown here for comparison. One is the scheme, termed as "equal gain scheme", where beamforming is not used and each relay just forwards its observations without weighting, and the other, termed as "centralized beamforming", where the optimal solution of (6) is used for weighting factors. It is interesting to observe that the performance of equal power transmission could result worse performance than direct transmission, which implies that it could be better not to use relay transmission without proper beamforming or power allocation. Furthermore, centralized power control can achieve better performance than direct transmission, as expected. And it can be observed that the proposed distributed method can have performance of $1 \mathrm{~dB}$ from that of the centralized one.

Figure 4 shows the diversity-multiplexing tradeoff for the three transmission schemes, direct transmission, selection relaying [2] and the proposed method. As expected, direct (or non cooperative) transmission can achieve a multiplexing gain of 1 as each orthogonal channel is only used by one user. Since orthogonal transmission is chosen, users cannot assist each other and hence there is no diversity gain. On the other hand, the selective relaying scheme can achieve a diversity gain of 2 as each copy of the transmitted message go through 2 independent channels and hence more robustness can be achieved. However such diversity gain is obtained at a price. It requires additional usage of bandwidth resource, i.e., time slots, for relaying. However, our proposed scheme can achieve the full diversity of $N_{r}+1$ while only requiring the same amount of bandwidth resource as the selection relay scheme.

\section{CONCLUSION}

In this paper, we have proposed a distributed algorithm which weighs the output of each relay node appropriately

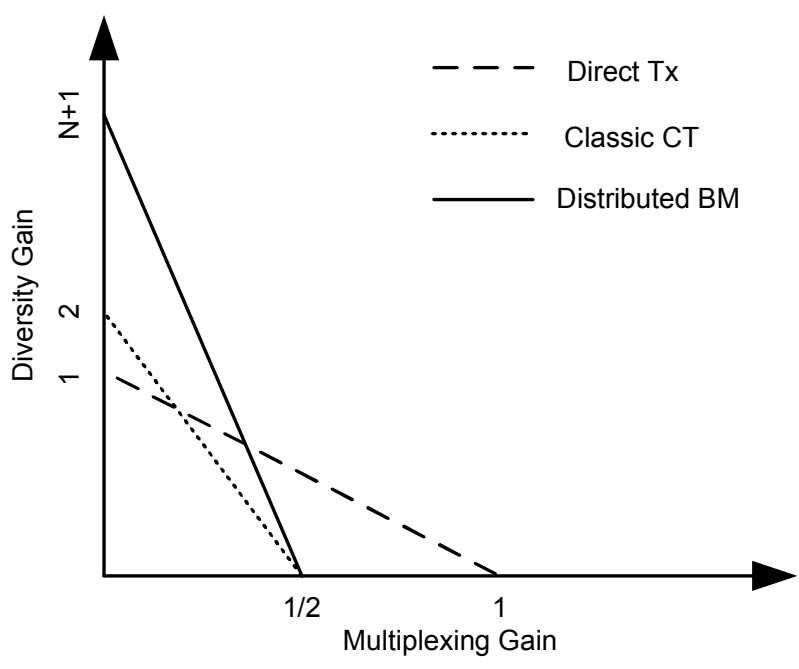

Fig. 4. Diversity-multiplexing tradeoff for the proposed beamforming scheme, selection cooperative schemes and non cooperative scheme

such that the quality of the combined received signal at the destination node is improved. We have also derived the outage probability of the algorithm as well as studied its outage behavior using Monte Carlo simulations. Due to the distributed nature of the proposed algorithm, it has motivated us to investigate how it can be extended and adopted in the design of cooperative networks. Furthermore, it will be an interesting future topic to study the design of a distributed beamformer with the power constraint at each relay.

\section{ACKNOWLEDGEMENT}

We would like to thank Ananthram Swami from U.S. Army Research Laboratory for helpful comments. And we also wish to thank the reviewers for their valuable comments.

\section{APPENDIX \\ OUTAGE PROBABILITY}

In this appendix, we derive the proof for theorem 1. To assist in the proof of the theorem, we first have following lemma.

Lemma 1: Let $\left|h_{i, d}\right|$ and $\left|h_{s, i}\right|$ be Rayleigh distributed random variables. For any positive finite integer $N_{r}$,

$$
\frac{\left(\sum_{i=1}^{N_{r}}\left|h_{s, i}\right|^{2}\left|h_{i, d}\right|^{2}\right)^{2}}{\sum_{i=1}^{N_{r}}\left|h_{s, i}\right|^{4}\left|h_{i, d}\right|^{2}} \leq \sum_{i=1}^{N_{r}}\left|h_{i, d}\right|^{2} .
$$

Proof of Lemma 1: Mathematical induction is used to prove this lemma. Define the left and right sides of (16) as functions of $N_{r}$,

$$
\begin{aligned}
& f(n)=\frac{\left(\sum_{i=1}^{n}\left|h_{s, i}\right|^{2}\left|h_{i, d}\right|^{2}\right)^{2}}{\sum_{i=1}^{n}\left|h_{s, i}\right|^{4}\left|h_{i, d}\right|^{2}}, \\
& g(n)=\sum_{i=1}^{n}\left|h_{i, d}\right|^{2}, \quad \text { for } \quad n \geq 1 .
\end{aligned}
$$

Hence proving Lemma 1 is equivalent to prove the the following

$$
f(n) \leq g(n), \quad \text { for } n \geq 1,
$$

which will be proved by using mathematical induction. 
1) When $n=1, f(1)=\left|h_{1, d}\right|^{2}=g(1)$.

2) Let $n=k$ and assume that

$$
f(k) \leq g(k) .
$$

To simplify the proof, further define $a_{k}=$ $\left(\sum_{i=1}^{k}\left|h_{s, i}\right|^{2}\left|h_{i, d}\right|^{2}\right)$ and $b_{k}=\sum_{i=1}^{k}\left|h_{s, i}\right|^{4}\left|h_{i, d}\right|^{2}$. Since $f(k) \leq g(k)$,

$$
a_{k}^{2}-b_{k} g(k) \leq 0
$$

For $n=k+1$,

$$
f(k+1)=\frac{\left(a_{k}+\left|h_{s, k+1}\right|^{2}\left|h_{k+1, d}\right|^{2}\right)^{2}}{b_{k}+\left|h_{s, k+1}\right|^{4}\left|h_{k+1, d}\right|^{2}},
$$

Hence, we have (21). Since $4 a_{k}^{2}-4 b_{k} g(k) \leq 0$, we have

$$
2 a_{k}\left|h_{s, k}\right|^{2}-g(k)\left|h_{s, k}\right|^{4}-b_{k} \leq 0
$$

which implies that

$$
f(k+1)-g(k+1) \leq 0
$$

Therefore, $f(n)-g(n) \leq 0$ if $f(1)-g(1) \leq 0$, and the lemma is proved.

Proof of Theorem 1: From (9), we can deduce that the outage probability of the system is as shown in (24), where $\theta=\frac{2^{2 R}-1}{\rho}$ and $\rho=\frac{P}{\sigma_{n}^{2}}$. Note that the second equality follows the high SNR assumption where $\sigma_{n}^{2} \approx 0$.

For large $N_{r}$, we have the following observations. Firstly,

$$
\frac{\sum_{i=1}^{N_{r}}\left|h_{i, d}\right|^{4}\left|h_{s, i}\right|^{2}}{\sum_{i=1}^{N_{r}}\left|h_{s, i}\right|^{4}\left|h_{i, d}\right|^{2}} \approx 1
$$

due to the fact that all channels are assumed i.i.d.. Furthermore,

$$
\frac{\left(\sum_{i=1}^{N_{r}}\left|h_{s, i}\right|^{2}\left|h_{i, d}\right|^{2}\right)^{2}}{\sum_{i=1}^{N_{r}}\left|h_{s, i}\right|^{4}\left|h_{i, d}\right|^{2}} \geq \frac{1}{N_{r}^{2}} \sum_{i=1}^{N_{r}}\left|h_{i, d}\right|^{2}
$$

Although we currently do not have the formal proof for this, simulations show that it is the case.

So we can have

$$
p(\mathcal{I}<R) \leq p\left(\left(\left|h_{s, d}\right|^{2}+\frac{1}{N_{r}^{2}} \sum_{i=1}^{N_{r}}\left|h_{i, d}\right|^{2}\right)<\alpha \theta\right)
$$

where $\alpha=2+P$.

Since it is assumed that the Rayleigh fading channels from $N_{r}$ source nodes to the destination node are i.i.d., $\left|h_{n, d}\right|^{2}$ are independent exponential variables with the decaying rate $\mu$. Hence $\sum_{n=0}^{N_{r}}\left|h_{n, d}\right|^{2}$ is Chi-square distribution with $2 N_{r}$ degrees of freedom. Define $x_{\Sigma}=\sum_{n=0}^{N_{r}}\left|h_{n, d}\right|^{2}$ and we have

$$
f_{x_{\Sigma}}\left(x_{\Sigma}\right)=C_{1} x_{\Sigma}^{N} e^{-x_{\Sigma}}
$$

where $C_{1}=\frac{1}{\left(N_{r}-1\right) !}$.
So the outage probability can be written as

$$
\begin{aligned}
p(\mathcal{I}< & R) \\
\leq & p\left(\left(\left|h_{s, d}\right|^{2}+\frac{1}{N_{r}^{2}} x_{\Sigma}\right)<\alpha \theta\right) \\
= & \int_{0}^{\alpha \theta} \int_{0}^{\alpha \theta-\frac{1}{N_{r}^{2}} x_{\Sigma}} e^{-\left|h_{s, d}\right|^{2}} d\left|h_{s, d}\right|^{2} f_{x_{\Sigma}}\left(x_{\Sigma}\right) d x_{\Sigma} \\
= & C_{1} \int_{0}^{\alpha \theta}\left(1-e^{-\left(\alpha \theta-\frac{1}{N_{r}^{2}} x_{\Sigma}\right)}\right) x_{\Sigma}^{N_{r}-1} e^{-x_{\Sigma}} d x_{\Sigma} \\
= & C_{1} \int_{0}^{\alpha \theta} x_{\Sigma}^{N_{r}-1} e^{-x_{\Sigma}} d x_{\Sigma}-C_{1} e^{-\alpha \theta} \\
& \int_{0}^{\alpha \theta} e^{-\left(1-\frac{1}{N_{r}^{2}}\right) x_{\Sigma}} x_{\Sigma}^{N_{r}-1} d x_{\Sigma}
\end{aligned}
$$

By using the exponential property, the outage probability can be simplified as

$$
\begin{aligned}
p(\mathcal{I}<R) & \\
\leq & C_{1} \int_{0}^{\alpha \theta} x_{\Sigma}^{N_{r}-1} e^{-x_{\Sigma}} d x_{\Sigma}-C_{1} e^{-\alpha \theta} \\
& \int_{0}^{\alpha \theta}\left(1-\left(1-\frac{1}{N_{r}^{2}}\right) x_{\Sigma}\right) x_{\Sigma}^{N_{r}-1} d x_{\Sigma} \\
= & {\left[1-e^{-\alpha \theta} \sum_{k=0}^{N_{r}-1} \frac{(\alpha \theta)^{k}}{k !}\right] } \\
- & C_{1}\left[\frac{1}{N_{r}}(\alpha \theta)_{r}^{N}-\frac{\left(1-\frac{1}{N_{r}^{2}}\right)}{N_{r}+1}(\alpha \theta)^{N_{r}+1}\right] \\
= & e^{-\alpha \theta} \sum_{k=N_{r}}^{\infty} \frac{(\alpha \theta)^{k}}{k !}-C_{1}\left[\frac{1}{N_{r}}(\alpha \theta)_{r}^{N}-\frac{\left(1-\frac{1}{N_{r}^{2}}\right)}{N_{r}+1}(\alpha \theta)^{N_{r}+1}\right] \\
\approx & \frac{N_{r}(\alpha \theta)^{N_{r}+1}}{\left(N_{r}+1\right) !}
\end{aligned}
$$

Using Lemma 1, we have the following inequality

$$
p(\mathcal{I}<R) \geq P\left(\left|h_{s, d}\right|^{2}+\sum_{i=1}^{N_{r}}\left|h_{i, d}\right|^{2}<\theta \alpha\right)
$$

Define $x_{\Delta}=\left|h_{s, d}\right|^{2}+\sum_{i=1}^{N_{r}}\left|h_{i, d}\right|^{2}$. From (28) we have the lower bound

$$
\begin{aligned}
p(\mathcal{I}<R) & \geq P\left(x_{\Delta}<\theta \alpha\right) \\
& =1-e^{-x_{\Delta}} \sum_{i=1}^{N_{r}} \frac{(\alpha \theta)^{i}}{i !} \\
& \approx 1-e^{-x_{\Delta}}\left[e^{-x_{\Delta}}-\sum_{i=N_{r}+1}^{\infty} \frac{(\alpha \theta)^{i}}{i !}\right] \\
& \approx \frac{(\alpha \theta)^{N_{r}+1}}{\left(N_{r}+1\right) !}
\end{aligned}
$$

Since both the upper and lower bounds of the outage probability have the same exponential equality, the theorem is proved.

\section{REFERENCES}

[1] J.G. Proakis, Digital Communications. McGraw Hill, 3rd ed., 1995.

[2] J. N. Laneman, D. N. C. Tse, and G. W. Wornell, "Cooperative diversity in wireless networks: efficient protocols and outage behavior," IEEE Trans. Inform. Theory, vol. 50, pp. 3062-3080, Dec. 2004. 


$$
\begin{aligned}
& f(k+1)-g(k+1)=\frac{\left(a_{k}+\left|h_{s, k+1}\right|^{2}\left|h_{k+1, d}\right|^{2}\right)^{2}}{b_{k}+\left|h_{s, k+1}\right|^{4}\left|h_{k+1, d}\right|^{2}}-g(k)-\left|h_{k+1, d}\right|^{2} \\
& =\frac{a_{k}^{2}+\left|h_{s, k+1}\right|^{4}\left|h_{k+1, d}\right|^{4}+2 a_{k}\left|h_{s, k+1}\right|^{2}\left|h_{k+1, d}\right|^{2}}{b_{k}+\left|h_{s, k+1}\right|^{4}\left|h_{k+1, d}\right|^{2}}-g(k)-\left|h_{k+1, d}\right|^{2} \\
& =\frac{\left(a_{k}^{2}-g(k) b_{k}\right)+\left|h_{k+1, d}\right|^{2}\left(2 a_{k}\left|h_{s, k+1}\right|^{2}-g(k)\left|h_{s, k+1}\right|^{4}-b_{k}\right)}{b_{k}+\left|h_{k+1, d}\right|^{2}\left|h_{s, k+1}\right|^{4}} \\
& p(\mathcal{I}<R) \approx p\left(\frac{P}{\sigma_{n}^{2}}\left(\left|h_{s, d}\right|^{2}+\frac{\left(\sum_{i=1}^{N_{r}}\left|h_{s, i}\right|^{2}\left|h_{i, d}\right|^{2}\right)^{2}}{\sum_{i=1}^{N_{r}}\left|h_{s, i}\right|^{4}\left|h_{i, d}\right|^{2}}\right)\left(2+P \frac{\sum_{i=1}^{N_{r}}\left|h_{s, i}\right|^{2}\left|h_{i, d}\right|^{4}}{\sum_{i=1}^{N_{r}}\left|h_{s, i}\right|^{4}\left|h_{i, d}\right|^{2}}\right)^{-1}<2^{2 R}-1\right) \\
& =p\left(\left(\left|h_{s, d}\right|^{2}+\frac{\left(\sum_{i=1}^{N_{r}}\left|h_{s, i}\right|^{2}\left|h_{i, d}\right|^{2}\right)^{2}}{\sum_{i=1}^{N_{r}}\left|h_{s, i}\right|^{4}\left|h_{i, d}\right|^{2}}\right)<\theta\left(2+P \frac{\sum_{i=1}^{N_{r}}\left|h_{s, i}\right|^{2}\left|h_{i, d}\right|^{4}}{\sum_{i=1}^{N_{r}}\left|h_{s, i}\right|^{4}\left|h_{i, d}\right|^{2}}\right)\right)
\end{aligned}
$$

[3] A. Sendonaris, E. Erkip, and B. Aazhang, "User cooperation diversitypart I: system description," IEEE Trans. Commun., vol. 51, pp. 19271938, Nov. 2003.

[4] P. Larsson and R. Hu, "Large-scale cooperative relaying network with optimal coherent combining under aggregate relay power constraints," in Proc. WG4, WWRF 8bis Meeting, Beijing, China, Feb. 2004.

[5] N. B. Mehta, A. F. Molisch, and J. Zhang, "Energy-efficient cooperative relaying over fading channels with simple relay selection," in Proc. GLOBECOM 2006, San Francisco, CA, 2006 (to appear).

[6] G. Barriac, R. Mudumbai, and U. Madhow, "Distributed beamforming for information transfer in sensor networks," in Proc. IPSN '04, Berkeley, CA, pp. 81-87, Apr. 2004.

[7] I. Hammerstrom, M. Kuhn, and A. Wittneben, "Impact of relay gain allocation on the performance of cooperative diversity networks," in Proc. VTC 2004 Fall, vol. 3, pp. 1815-1819, Sept. 2004.

[8] X. Deng and A. M. Haimovich, "Power allocation for cooperative relaying in wireless networks," IEEE Commun. Lett., vol. 9, pp. 994996, Nov. 2005.

[9] Y. Zhao, R. Adve, and T. J. Lim, "Improving amplify-and-forward relay networks: optimal power allocation versus selection," IEEE Trans. Wireless Commun (to appear).

[10] A. F. Dana and B. Hassibi, "On the power efficiency of sensory and ad hoc wireless networks," IEEE Trans. Inform. Theory, vol. 52, pp. 28902914, July 2006.

[11] L. Zheng and D. N. C. Tse, "Diversity and multiplexing: a fundamental tradeoff in multiple-antenna channels," IEEE Trans. Inform. Theory, vol. 49, pp. 1073-1096, May 2003.

[12] A. Bletsas, H. Shin and M. Z. Win, "Outage optimality of opportunistic amplify-and-forward relaying," IEEE Commun. Lett., vol. 11, pp. 261C263, Mar. 2007. 